\title{
Nonreciprocal and Directional Wave Propagation in a Two-Dimensional Lattice with Bilinear Properties
}

\author{
Zhaocheng Lu \\ E-mail: zhaocheng.lu@rutgers.edu \\ and Andrew N. Norris \\ Department of Mechanical and Aerospace Engineering, \\ Rutgers University, Piscataway, NJ 08854-8058 (USA)
}

(Dated: May 21, 2021)

\begin{abstract}
A passive method of realizing nonreciprocal wave propagation in a two-dimensional (2D) lattice is proposed, using bilinear springs combined with the necessary spatial asymmetry to provide a stable and strong departure from reciprocity. The bilinear property is unique among nonlinear mechanisms in that it is independent of amplitude but sensitive to the sign of the wave motion; the 2D setup allows the flexibility of generating spatial asymmetry at both small and large scales. The starting point is a linear $2 \mathrm{D}$ monatomic spring-mass lattice with strong directionally dependent wave propagation. The source and receiver are aligned so that there is virtually no direct wave transmission between them. Adding a region of bilinearity combined with spatial asymmetry that is not in the direct path between the source and receiver causes signal transmission via nonreciprocal scattering. A variety of spatially asymmetric bilinear configurations are considered, ranging from compact modulations confined within the unit cell to extended ones over the whole section, to obtain different dynamic nonreciprocal effects. Simulations illustrate how the combination of bilinearity and spatial asymmetry ensures a passive amplitude-independent nonreciprocal 2D system for a variety of different excitations.
\end{abstract}




\section{INTRODUCTION}

Reciprocity is a fundamental physical principle of wave motion that guarantees symmetric wave transmission between a source and a receiver. Interchanging the positions of source and receiver in a reciprocal medium results in the same signal. Overcoming this restriction can lead to comprehensive control of wave propagation [1]. Breaking reciprocity in one-dimensional (1D) structures can be achieved in many different ways, either using external energy to modulate the system properties (active methods) or introducing nonlinearity with spatial asymmetry (passive methods). The energetic approaches fall into two types of methods: introducing moving parts or circulating flows in the propagation medium [2, 3], and performing spatial-temporal modulations of the system properties [4-10]. The passive methods typically make use of various nonlinear mechanical properties and the necessary ingredient of spatial asymmetry [11-17]. Many researchers have successfully realized breaking reciprocity in 1D domain via active and passive approaches, demonstrating effects such as one-way acoustic and elastic wave propagation [1, 4, 8, 15, 16, 18], asymmetric energy transfer [1, 10, 13, 17] and nonreciprocal phase shift [1, 3].

However in 2D space only the active nonreciprocal systems have been considered. Of the two principle methods used the first is nonreciprocal topologically protected edge states (TPESs) that appear at the interface between two topologically inequivalent insulators. For nonreciprocal TPESs, unidirectional wave propagation along an interface is supported because one of the two topologies is created by breaking time-reversal symmetry. Active components, which are able to modulate the physical properties in time, are typically used to break time-reversal symmetry. Examples are experimentally realized lattices of gyroscopes [19], lattices of acoustic circulators with moving fluid involved [20], and theoretical studies of chiral edge modes in hexagonal and square gyroscopic lattices [21]. The second active method uses space-time modulation, as in 1D. For example, nonreciprocal transmission of Rayleigh surface waves with one-way mode conversion can be realized in a continuous 2D semi-infinite medium bound with an array of space-time modulated spring-mass oscillators [22]. Both the topological insulators and the semi-infinite medium with complex interface are active nonreciprocal systems which require external energy input, and as a consequence they are potentially unstable.

Here we concentrate on a passive approach to breaking wave reciprocity in a specific $2 \mathrm{D}$ 
system. We take advantage of the fact that 2D periodic structures usually exhibit directional wave propagation when some requirements, e.g. structural properties and wave frequency range, are satisfied [23 25]. This phenomenon indicates the existence of propagation-free region in those structures, which inspires us to come up with a strategy of realizing the customized nonreciprocal behavior: wave transmission in the propagation-free region with opposite displacements. Material nonlinearity combined with spatial asymmetry can be added to the $2 \mathrm{D}$ periodic structure, redirecting the signals to a propagation-free region via scattering from the asymmetric nonlinear section. Similar idea is found in some previous works, where cubic nonlinearity has been used in 2D lattices to introduce tunable wave directionality [24, 25]. However, these systems are heavily dependent on excitation amplitude, and are still reciprocal because of the lack of the necessary and essential ingredient of spatial asymmetry for breaking reciprocity. Instead, we use bilinearity, which is independent of amplitude but sensitive to the sign of wave motion, to generate a stable and strong nonlinearity. Besides, taking advantage of the $2 \mathrm{D}$ condition, we can design a variety of spatially asymmetric configurations of bilinearity at both small and large scales to obtain different scattering results before and after interchanging the positions of source and receiver, and therefore passively break reciprocity. This paper also provides a theoretical support for future experimental efforts.

The outline of the paper is as follows. Section II discusses the physical structure of the linear 2D monatomic lattice and the directional wave propagation properties. The design principle of breaking reciprocity by adding a bilinear section with spatial asymmetry to the lattice is introduced in Sect. [II]. Based on the flexibility of generating spatial asymmetry in the $2 \mathrm{D}$ lattice, we start with micro-modulations within each unit cell of the bilinear section in Sect. IV] Interpretation and amplitude independence of the nonreciprocal effect are demonstrated, and several simulation arrangements are considered. An additional design approach using macro-modulations of the whole bilinear section in its entirety is then discussed in Sect. V. Section VI concludes the paper. 


\section{2D MONATOMIC LATTICE}

\section{Equations of motion}

A 2D monatomic lattice is modeled as an array of equal masses interconnected by shear springs, as shown in Fig. 1. Transverse (into- and out-of-plane) displacement is the single degree of freedom describing the motion of each mass, and springs are assumed to act in shear with a force which is related to the relative displacements of neighboring masses. The unit cell therefore consists of a block with mass and a mass-less " +" shape structure (no force between them) with thickness, width and length $d \rightarrow 0$ as Fig. 1 shows. The lengthless "+" shape structure is able to transfer force between neighboring masses, leading to the transverse wave propagation while neglecting rotational motion. Two states (" +" shape up and down) exist in this 2D lattice, guaranteeing the spatial symmetry of the lattice.
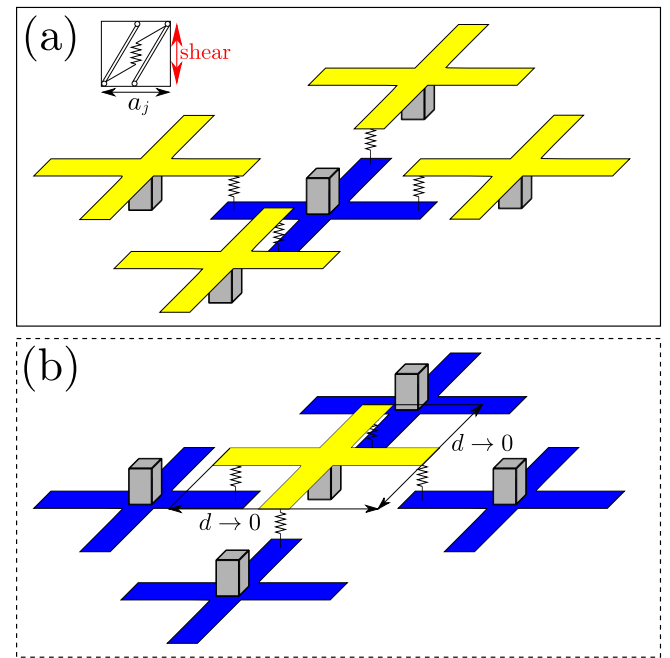

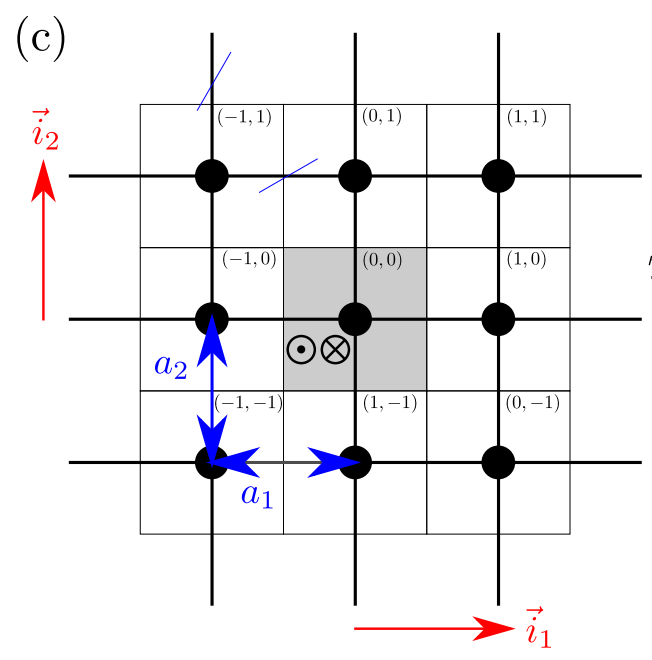
unit cell at $\left(n_{1}, n_{2}\right)$

FIG. 1: 2D monatomic lattice of identical masses connected by shear springs. (a) and (b) depict the physical structure of a unit cell in the monatomic lattice, which consists of a block with mass and a mass-less " +" shape structure of size $d \rightarrow 0$ introducing the in-plane transverse wave propagation; (a) stands for the state of "+" shape up and (b) down. The insert in (a) is a schematic of a shear spring with structural length $a_{j}, j=1,2$. (c) shows the reference system; the mass displacement is in the transverse direction (into- and out-of-plane) only. In order to eliminate reflections from the boundaries for numerical simulations, the finite lattice is surrounded by perfectly matched layers (PMLs), see Appendix A for details. 
The periodicity of the lattice is defined by orthogonal lattice vectors $a_{1} \vec{i}_{1}$ and $a_{2} \overrightarrow{i_{2}}$ in the horizontal and vertical direction, respectively. The mass at coordinates $\left(n_{1}, n_{2}\right)$, shown in grey box at the center in Fig. 1(c), $\vec{r}_{\left(n_{1}, n_{2}\right)}=n_{1} a_{1} \vec{i}_{1}+n_{2} a_{2} \overrightarrow{i_{2}}$, satisfies the equilibrium equation (supposing that the force exerted by the spring is much greater than gravity and neglecting rotational motion)

$$
m \ddot{u}=\sum_{j=1}^{2}\left[\kappa_{j}^{-} \Delta u_{j}^{-}+\kappa_{j}^{+} \Delta u_{j}^{+}\right],
$$

where $u=u_{\left(n_{1}, n_{2}\right)}$, and

$$
\kappa_{j}^{-}=\kappa_{j\left(n_{1}, n_{2}\right)}, \kappa_{j}^{+}=\kappa_{j}\left(n_{1}+\delta_{j 1}, n_{2}+\delta_{j 2}\right),
$$

with $j=1$ and 2 denoting stiffnesses of the springs located along the horizontal and vertical direction, respectively, and

$$
\Delta u_{j}^{ \pm}=u_{\left(n_{1} \pm \delta_{j 1}, n_{2} \pm \delta_{j 2}\right)}-u_{\left(n_{1}, n_{2}\right)}
$$

representing the relative transverse displacement of two adjacent masses.

\section{Wave directivity of a linear lattice}

The dispersion relation between the frequency $\omega$ and the wave vector $\vec{k}=k_{1} \vec{i}_{1}+k_{2} \overrightarrow{i_{2}}$ plays an important role in deciding the wave propagation in the lattice. Setting the transverse displacement of the neighboring masses as

$$
u_{\left(n_{1} \pm 1, n_{2} \pm 1\right)}=u_{\left(n_{1}, n_{2}\right)} e^{i\left( \pm k_{1} a_{1} \pm k_{2} a_{2}\right)}
$$

and assuming time dependence $e^{-i \omega t}$ yields the dispersion relation

$$
\omega^{2}=\frac{2}{m}\left[\kappa_{1}\left(1-\cos k_{1} a_{1}\right)+\kappa_{2}\left(1-\cos k_{2} a_{2}\right)\right] .
$$

Based on Eq. (5), we plot the dispersion surface and the corresponding isofrequency contours for the monatomic lattice in Fig. 2(a) and (b).

The group velocity

$$
\vec{c}_{g}=\frac{\partial \omega}{\partial k_{1}} \vec{i}_{1}+\frac{\partial \omega}{\partial k_{2}} \vec{i}_{2} \equiv c_{g 1} \vec{i}_{1}+c_{g 2} \vec{i}_{2}
$$

where

$$
c_{g j}=\frac{a_{j} \kappa_{j}}{m \omega} \sin k_{j} a_{j}, \quad j=1,2
$$



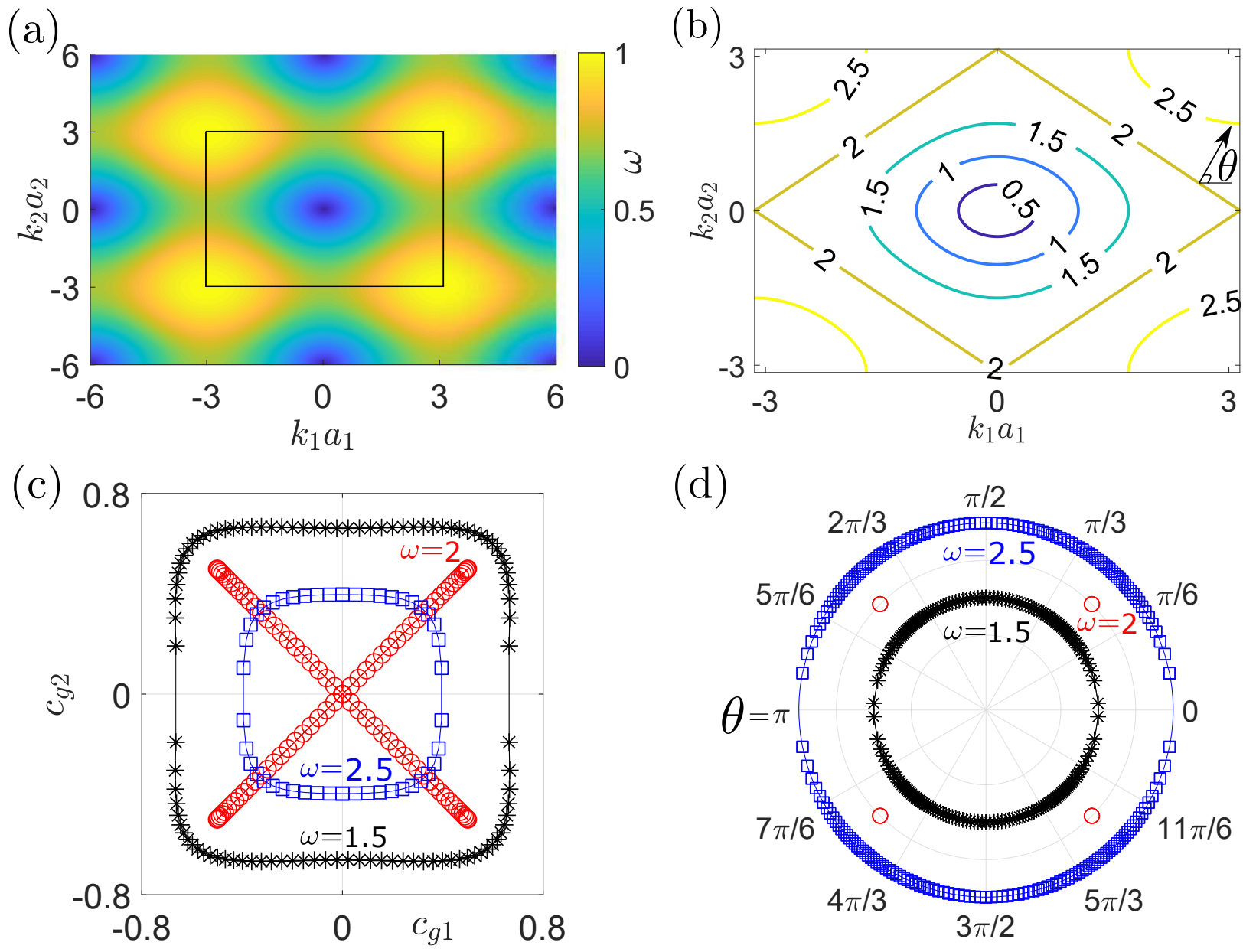

(d)

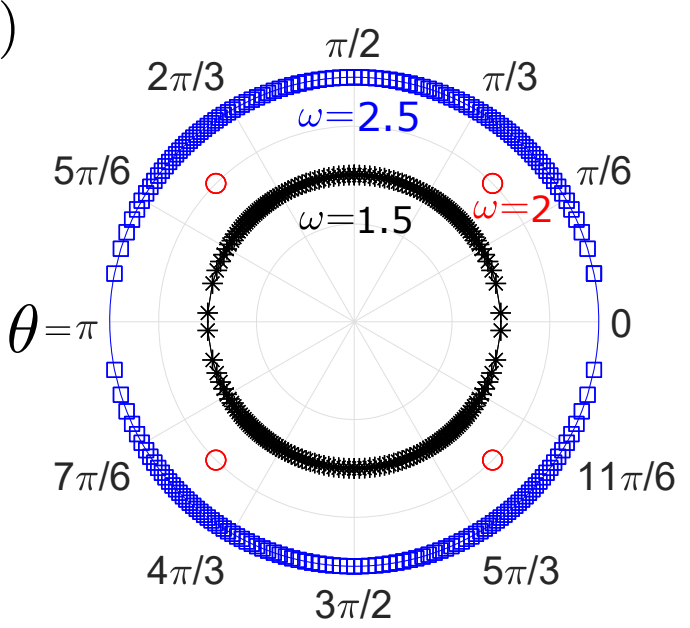

FIG. 2: Tools for the analysis of wave directivity. (a) Dispersion surface. (b) Isofrequency contours of frequency distribution within the first Brillouin zone (black rectangle) in (a); $\theta$ defines the direction of wave energy propagation. (c) Group velocity (50 data points per plot). (d) Directions of wave energy propagation (200 data points per plot). Here $a_{1}=a_{2}=1 \mathrm{~m}, \kappa_{1}=\kappa_{2}=1 \mathrm{~N} / \mathrm{m}$, $m=1 \mathrm{~kg}$, and frequency $\omega$ is measured in $\mathrm{rad} / \mathrm{s}$.

is depicted in Fig. 2(c), only the case of wave traveling in all directions in which has a closed contour.

The angle formed between normal to the isofrequency contour and the horizontal axis in Fig. 2(b) is denoted as $\theta$, which defines the direction of wave energy propagation at the corresponding wavenumber pair. The propagation directions are shown in the polar plot Fig. 2(d), and the expression of propagating angle follows from Eq. (7)

$$
\theta=\tan ^{-1} \frac{a_{2} \kappa_{2} \sin k_{2} a_{2}}{a_{1} \kappa_{1} \sin k_{1} a_{1}}
$$


where the values of $k_{1}$ and $k_{2}$ are specified by the isofrequency contour of Fig. 2(b) for a selected frequency $\omega$.

\section{DESIGN OF NONRECIPROCAL 2D MONATOMIC LATTICE}

The design for breaking reciprocity takes advantage of the directional nature of wave propagation in the 2D lattice. The idea, illustrated in Fig. 3, is as follows. In the linear case, directional wave propagation from source in Fig. 3(a) cannot be detected at receiver $(\mathrm{A} \rightarrow \mathrm{B}$ or $\mathrm{B} \rightarrow \mathrm{A})$ because the latter is, by choice, located in a propagation-free region relative to the source. Then by introducing bilinearity with spatial asymmetry in a place that a wave can reach from the source, see Fig. 3(b), a signal will travel from the source to the receiver via scattering from the bilinear section. A different response will be observed after interchanging the positions of source and receiver, see Fig. 3(c), due to nonreciprocity achieved by the combination of bilinearity and spatial asymmetry.
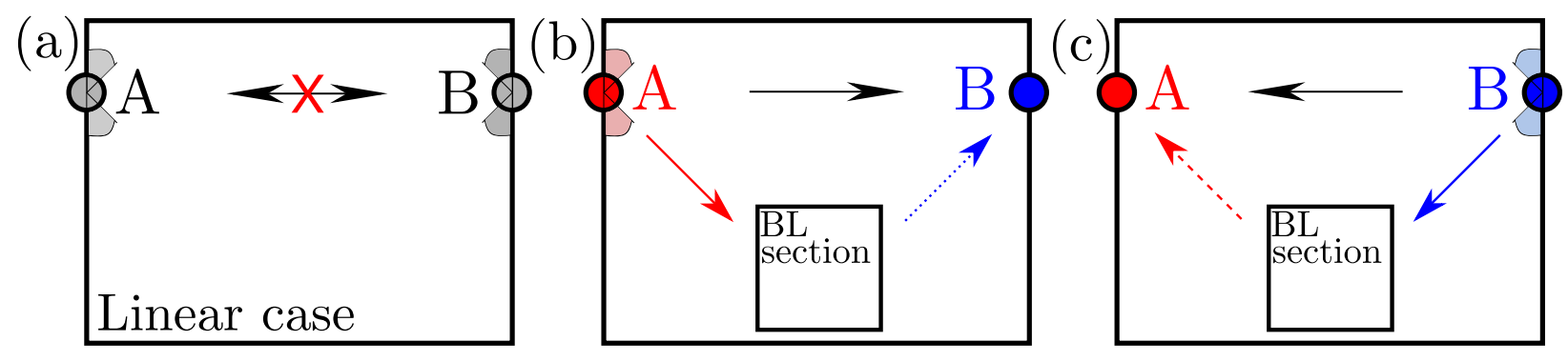

FIG. 3: Schematic diagram of the design for a nonreciprocal 2D monatomic lattice. (a) depicts directionally-limited wave propagation in the linear lattice, where the propagating range is indicated by shaded angles; receiver at B (A) is located in the propagation-free region relative to source at A (B). The bilinear section (square box) combined with spatial asymmetry is then introduced in a place that a wave can reach from the source; (b) and (c) illustrate that a signal will travel from the source to the receiver via scattering from the asymmetric bilinear section, and interchanging the positions of source and receiver results in different responses because of the spatial asymmetry in the bilinear region (not shown).

Spatial asymmetry is a necessary ingredient for breaking reciprocity passively in bilinear medium. Given the 2D condition, we have the flexibility of combining bilinearity with various spatially asymmetric setups. Here we start with the modulations confined within each 
unit cell of the bilinear section, achieving the asymmetric arrangements in the microscopic manner. Then we apply additional modulations of asymmetry to the whole bilinear section as its entirety from the macro perspective.

\section{MICRO-MODULATION: SPATIAL ASYMMETRY OF UNIT CELL}

The system remains reciprocal despite the bilinearity introduced if it is spatially symmetric. Here we discuss different ways of introducing micro-structural spatial asymmetry within the unit cell of nonlinear section and therefore break reciprocity.

\section{Stiffness and structural asymmetry}

To start with, spatial symmetry for a unit cell of a linear 2D monatomic lattice indicates that the same resultant shear force, $F_{j}^{ \pm}=\kappa_{j}^{ \pm} \Delta u_{j}^{ \pm}$, can be obtained given the same relative displacement between the unit cell and its nearest neighbors, $\Delta u_{j}^{ \pm}$,

$$
\left\{\begin{array}{l}
\Delta u_{j}^{-}=\Delta u_{j}^{+} \\
\kappa_{j}^{-}=\kappa_{j}^{+}
\end{array} \Rightarrow F_{j}^{-}=F_{j}^{+},\right.
$$

$j=1$ when we consider the neighboring unit cells in horizontal direction, and $j=2$ in vertical direction.

Spatial symmetry still holds in the unit cell when all linear springs are replaced by identical bilinear springs (labelled by $\nearrow$ and the corresponding stiffness written as $\kappa \nearrow$ ) as shown in Fig. 4(a). Although the bilinear spring has different stiffnesses when it is compressed and stretched, two horizontally or vertically adjacent bilinear springs are always in the same condition given the same relative displacement, such that

$$
\left\{\begin{array}{l}
\Delta u_{j}^{-}=\Delta u_{j}^{+} \\
\kappa_{j}^{-}=\kappa_{j}^{+} \equiv \kappa_{(c \text { or } t) \nearrow}
\end{array} \quad \Rightarrow F_{j}^{-}=F_{j}^{+},\right.
$$

where $\kappa_{c} \nearrow$ stands for the compressive stiffness of the bilinear spring labelled by $\nearrow$, and $\kappa_{t} \nearrow$ the tensile stiffness.

One way to generate spatial asymmetry within the unit cell is to modify the stiffness property of the bilinear springs in an alternating manner. In Fig. 4(c), we replace the 
(a)

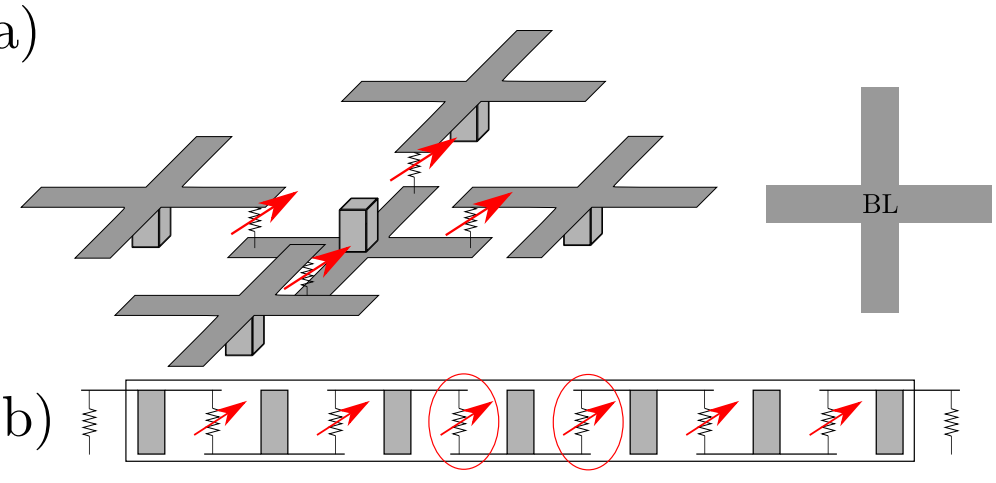

(c)

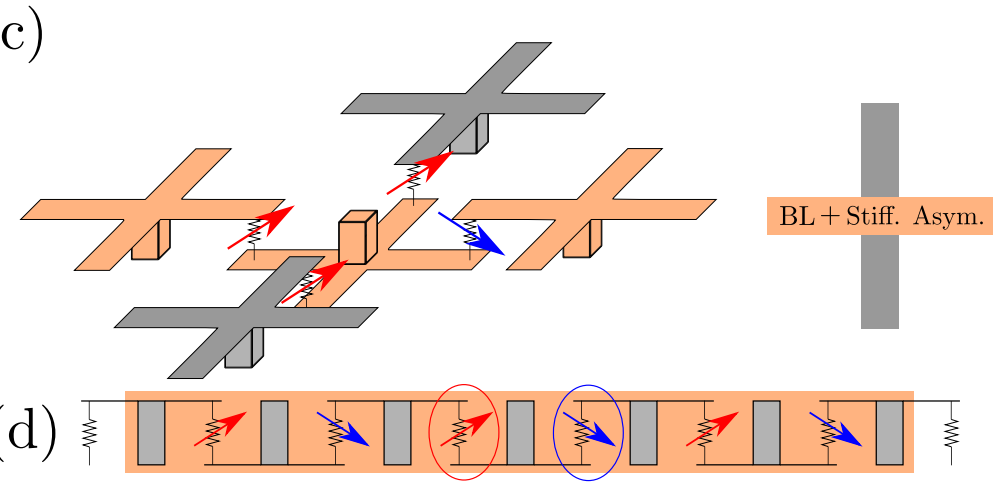

(e)

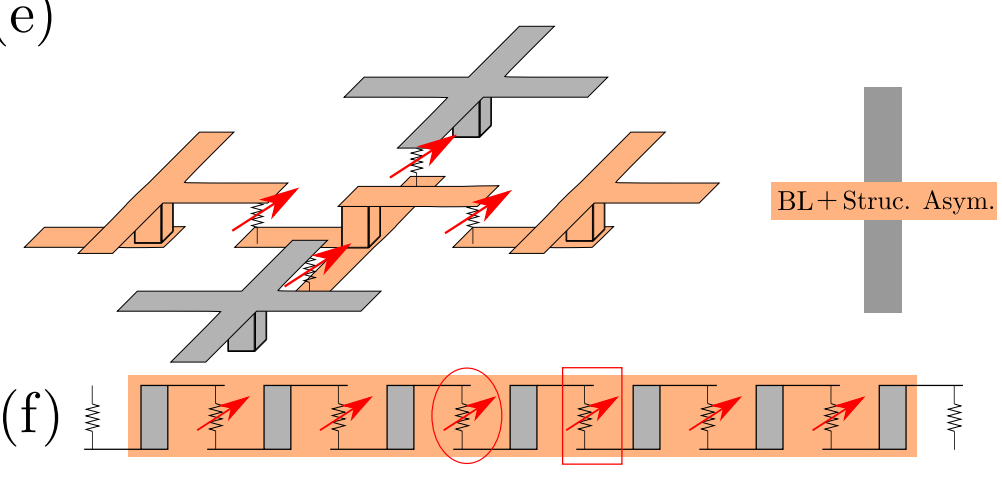

FIG. 4: Schematic of spatially symmetric and asymmetric setups. (a) shows the physical structure of a unit cell in the bilinear section connecting its nearest neighbors by identical bilinear springs, but without spatial asymmetry. (c) and (e) are two functionally identical asymmetric setups, with the asymmetric modulation in horizontal direction only. The shaded bars on the right illustrate the spatially symmetric or asymmetric conditions in corresponding directions. We alternate the bilinear spring connected to the unit cell in (c), leading to bilinear stiffness asymmetry; in (e), one leg in " +" shape structure is moved to the opposite position, resulting in structural asymmetry of the unit cell. (b), (d) and (f) depict chains (in horizontal direction) of the unit cells consisting of the corresponding bilinear setups, which intuitively show the compressive/stretched conditions of the bilinear springs and assist in the analysis of spatially asymmetric systems. 


\begin{tabular}{||c|c|c||}
\hline Bilinear Spring Label & Comp. Stiff. & Tens. Stiff. \\
\hline \hline$\nearrow$ & $\kappa_{c \nearrow}=\kappa-\Delta \kappa_{-}$ & $\kappa_{t} \nearrow=\kappa+\Delta \kappa_{+}$ \\
\hline$\searrow$ & $\kappa_{c \searrow}=\kappa+\Delta \kappa_{+}$ & $\kappa_{t \searrow}=\kappa-\Delta \kappa_{-}$ \\
\hline
\end{tabular}

TABLE I: Stiffness properties of bilinear springs. $\kappa$ is the linear stiffness and $\Delta \kappa_{ \pm}$are the devia-

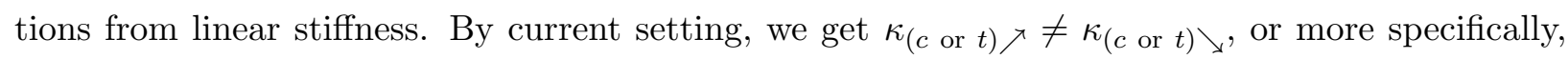
$\kappa_{(c \text { or } t) \nearrow}=\kappa_{(t \text { or } c) \searrow}$.

bilinear spring on the right of $\nearrow$ label with that of $\searrow$ label (therefore, we have $j=1$ for the following relevant equations, representing the replacement in the horizontal direction); these two springs exhibit different stiffnesses when compressed and stretched, see Table I. This modulation generates different resultant shear forces despite given the same relative displacement between the unit cell and its nearest neighbors, i.e.,

$$
\left\{\begin{array}{l}
\Delta u_{1}^{-}=\Delta u_{1}^{+} \\
\kappa_{1}^{-} \equiv \kappa_{(c \text { or } t)} \neq \kappa_{1}^{+} \equiv \kappa_{(c \text { or } t) \searrow}
\end{array} \quad \Rightarrow F_{1}^{-} \neq F_{1}^{+} .\right.
$$

The alternative micro-modulation is depicted in Fig. 4(e), one leg in " +" shape structure of the unit cell is moved to the opposite position, up or down. Without replacing any bilinear spring, the asymmetric structure results in the opposite conditions for two adjacent bilinear springs in horizontal direction $(j=1)$, one is compressed and the other stretched, given the same relative displacement, and therefore different resultant shear forces,

$$
\left\{\begin{array}{l}
\Delta u_{1}^{-}=\Delta u_{1}^{+} \\
\kappa_{1}^{-} \equiv \kappa_{(c \text { or } t) \nearrow} \neq \kappa_{1}^{+} \equiv \kappa_{(t \text { or } c) \nearrow}
\end{array} \Rightarrow F_{1}^{-} \neq F_{1}^{+} .\right.
$$

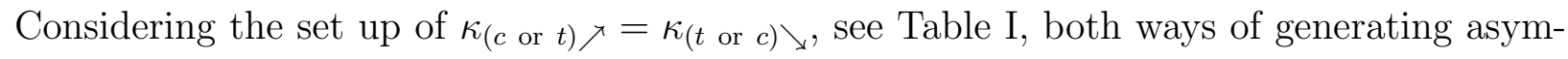
metry within the unit cell are functionally identical.

From the horizontal chain's perspective, the spring condition and resultant shear force for the symmetric and asymmetric (reciprocal and nonreciprocal) unit cell are self-evident, see Fig. 4(b), (d) and (f). In reciprocal case, Fig. 4(b), for any two adjacent bilinear springs, the same relative displacement leads to the same condition for them and the same resultant shear force. For our first strategy of generating spatial asymmetry shown in Fig. 4(d), the bilinear springs are reversed alternately; even though the same relative displacement results in the same condition for the adjacent reversal bilinear springs, different resultant shear 
forces are obtained. The structural asymmetry can be achieved using the second strategy as shown in Fig. $4(\mathrm{f})$; the same relative displacement results in the opposite conditions for two adjacent identical bilinear springs and therefore different resultant shear forces.

\section{Numerical experiments}

From now on, let us discuss the numerical simulations of wave propagation in the 2D monatomic lattice. To generate incident wave, a continuous displacement restriction is applied to source position,

$$
u=\mathcal{H}(t) U \sin \omega t
$$

where $U$ is input amplitude, $\omega$ is the excitation frequency, and $\mathcal{H}$ represents the Heaviside function. The positions A and B, which applied to place the source and receiver, are aligned so that there is virtually no direct wave transmission between them when strong directionally dependent wave propagates.
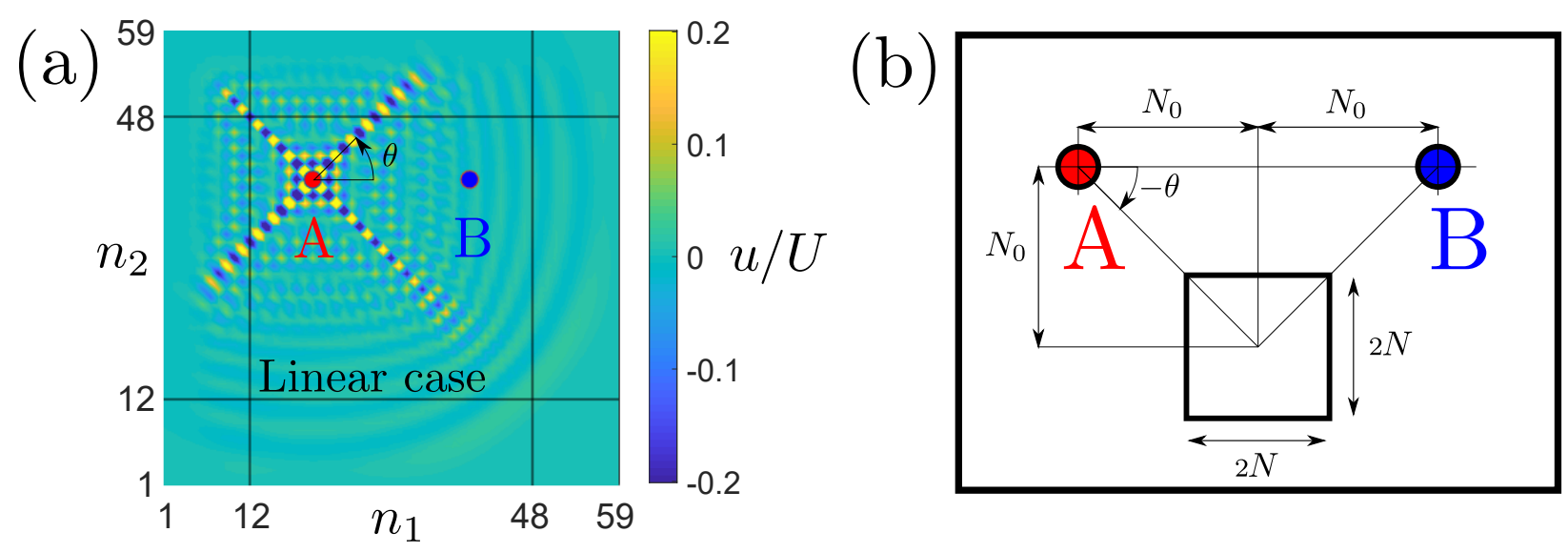

FIG. 5: Incident wave and simulation setup. (a) shows the incident wave directionally propagating in a pure linear lattice, generated by a continuous excitation, see Eq. (13). (b) illustrates the bilinear section, assumed to be square with sides of $2 N$ bilinear springs in length; the positions A and B (used to locate the source and receiver) are equally distant from the center of the bilinear section with $N_{0}$ springs in both horizontal and vertical directions.

Assuming that $a_{1}=a_{2}=1 \mathrm{~m}, \kappa_{1}=\kappa_{2}=1 \mathrm{~N} / \mathrm{m}, m=1 \mathrm{~kg}$ for model setup, and $U=1$ $\mathrm{m}, \omega=2 \mathrm{rad} / \mathrm{s}$ for excitation, we can obtain directional wave propagation in four discrete directions with angle $\theta=\frac{n \pi}{4}, n=1,3,5,7$, refer to Fig. 2 (c) and (d). Simulation result of 
this directional wave propagation in a pure linear lattice is shown in Fig. 5(a).

The location of an introduced bilinear section is then chosen so that the mentioned directional propagating wave reaches it. We take the bilinear section as a square with $2 N \times 2 N$ bilinear springs, see Fig. 5(b), whose center at angel $\theta=-\frac{\pi}{4}$ relative to the position $\mathrm{A}$ and $\theta=\frac{5 \pi}{4}$ for B. Therefore, the positions A and B are equally distant by $N_{0}$ springs from the center of the bilinear section.

Here we set the number of springs $N_{0}=10$ specifying the source and receiver positions, and $N=8$ the bilinear section size. Extreme bilinearity guarantees the significantly nonreciprocal results, requiring drastic difference between tensile and compressive stiffness, e.g. $\Delta \kappa_{-} \ll \Delta \kappa_{+}$, see Table $\amalg$ for details of stiffnesses selection.

\begin{tabular}{||c|c|c|c|c|c|c||}
\hline$\kappa_{1}=\kappa_{2}=\kappa$ & $\Delta \kappa_{-}$ & $\Delta \kappa_{+}$ & $\kappa_{c} \nearrow$ & $\kappa_{t} \nearrow$ & $\kappa_{c \searrow}$ & $\kappa_{t} \searrow$ \\
\hline \hline 1 & 0.875 & 10 & 0.125 & 11 & 11 & 0.125 \\
\hline
\end{tabular}

TABLE II: The stiffness of linear and bilinear springs. All linear springs are identical. The expressions of bilinear springs can be found in Table I with $\kappa_{c} \nearrow \ll \kappa_{t} \nearrow, \kappa_{c} \searrow \gg \kappa_{t} \searrow$ and $\kappa_{(c \text { or } t) \nearrow}=$

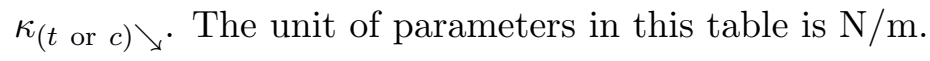

\section{Nonreciprocity}

Three different setups are tested, and the simulation results illustrate that combination of bilinearity and spatial asymmetry is able to break reciprocity.

Case 1: Pure linear. A pure linear setup is shown in Fig. 6(a), and the resultant dynamic profiles at the receivers are in Fig. 6(b). The overlapping low-amplitude results observed before and after interchanging the positions of source and receiver, typically below $10 \%$ of the excitation amplitude, not only indicate that the receivers are located at the propagation-free zone relative to the sources but illustrate the reciprocity.

Case 2: Bilinearity only. In Fig. 6(c), we introduce a bilinear section to the place where directional wave propagation reaches, refer to Fig. 5(b). However, the overlapping low-amplitude dynamic profiles in Fig. 6(d) indicate that a bilinear section without spatial asymmetry can not break reciprocity.

Case 3: Bilinearity + spatial asymmetry. We combine bilinearity with spatial asymmetry and arrange this setup along the horizontal direction only within the bilinear 

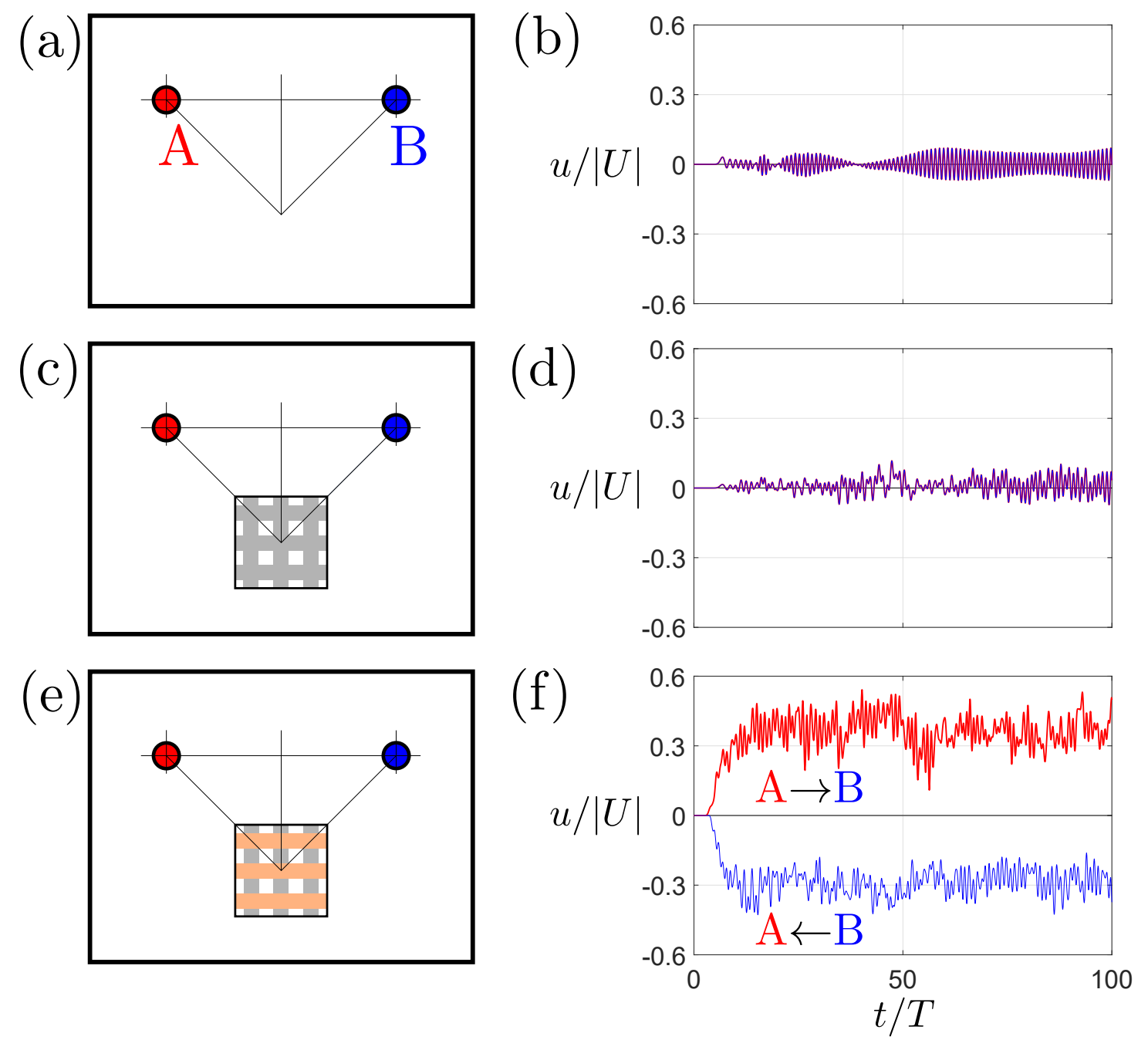

FIG. 6: Simulation results. The left column depicts three different simulation setups, refer to Fig. 4 and 5(b); the right one records the corresponding dynamic profiles of receivers, the normalized displacement $u /|U|$ against the number of excitation period. (a) shows the pure linear setup and (b) shows its reciprocal results with the overlapping low-amplitude dynamic profiles. The setup in (c) contains a squared section with the bilinearity in both horizontal and vertical directions; the overlapping dynamic results in (d) again indicates the reciprocity. Spatial asymmetry is introduced in (e), and the combination of bilinearity and spatial asymmetry is arranged in horizontal directions only; (f) shows that the dynamic profiles of receivers have the opposite signs of steady shifts with positive one for the source located at position $\mathrm{A}$ and negative one at $\mathrm{B}$. 
section as shown in Fig. 6(e). The resultant dynamic profiles have the opposite shifts see Fig. 6(f): a positive shift is obtained when the incidence comes from position A, and a negative one from B. Even though the normalized amplitude is still relatively low, roughly between 30 and 60\%, we lay more emphasis on the nonreciprocal dynamic behaviors with steady opposite shifts.

\section{Interpretation of nonreciprocal effects}

The reason for the opposite-sign dynamic profiles is discussed. Based on our previous explorations in the 1D bilinear spring-mass chain system [15], we conclude that the relation between the compressive and tensile stiffness of bilinear spring and the asymmetric arrangements of bilinearity strongly influence the dynamic behavior, which can also help us understand the simulation results in current $2 \mathrm{D}$ case.

(a)
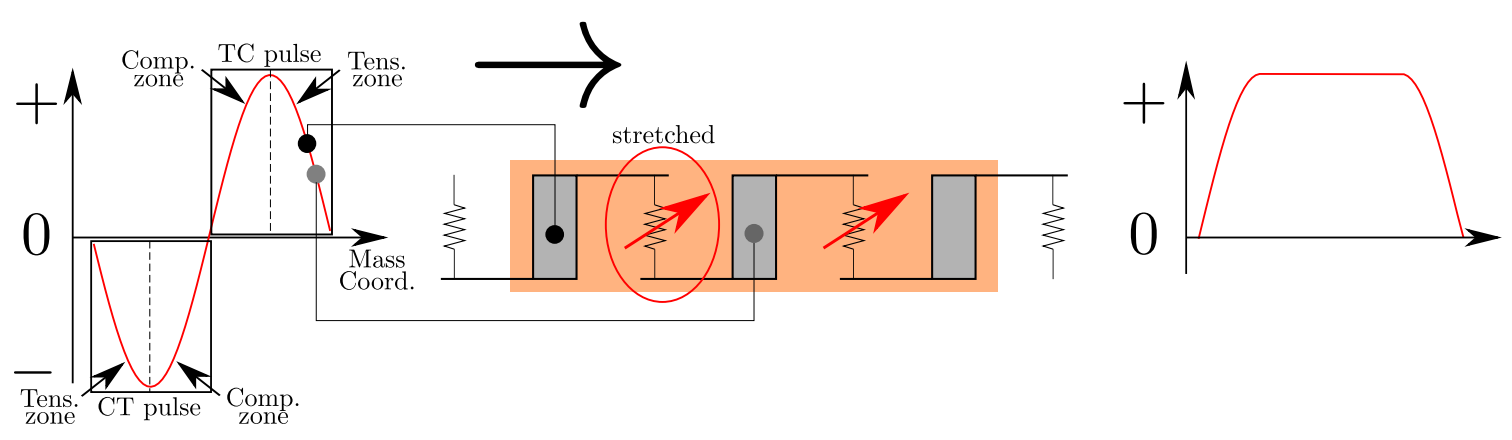

(b)

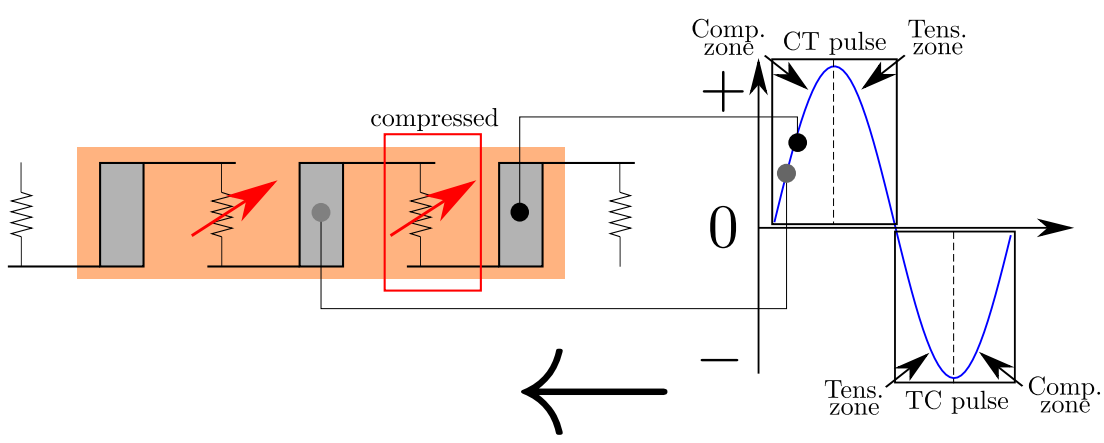

FIG. 7: Nonreciprocal wave propagation in an asymmetric bilinear chain. (a) and (b) show the single-cycle incident waves and the transmitted waves for incidence from left and right, respectively. The chains in shade sections contain structurally asymmetric unit cells connected by identical bilinear springs, see Fig. $4(f)$.

Figure 7 shows the propagation of a single-cycle incident wave in a spring-mass chain 
system from the opposite directions. Two types of pulses are formed when the incidence enters the bilinear section; a dashed line divides a pulse into different parts each of which relates to bilinear springs in the same state, compression or tension. Therefore, TC pulse (CT pulse) indicates a pulse with a tensile zone followed by a compressive zone (a compressive zone is followed by a tensile zone).

Since a tensile wave travels with a higher speed than a compressive one under current stiffness $\operatorname{setup}\left(\kappa_{c} \nearrow \ll \kappa_{t} \nearrow\right)$, we expect an increase in distance between the tensile and compressive zones and the generation of a zero deformation zone (the horizontal region with nearly constant positive displacement) for a TC pulse; in terms of a CT pulse, we expect that the faster tensile wave front catches up with the slower compressive one and then changes the pulse type to TC one. Table III concludes these two processes. Consequently, the same incident wave from the opposite directions results in different transmitted pulses with opposite displacement.

\begin{tabular}{|c|c|c|c|c|}
\hline Pulse Type & Property & Stiffness & Wave Speed & Result \\
\hline TC pulse & $\begin{array}{l}\text { Tens. zone precedes } \\
\text { Comp. zone }\end{array}$ & \multirow{2}{*}{$\kappa_{c} \nearrow \ll \kappa_{t} \nearrow$} & \multirow{2}{*}{$\begin{array}{c}\text { Comp. } \ll \text { Tens. } \\
\text { wave }\end{array}$} & $\begin{array}{l}\text { TC pulse with } \\
\text { zero deformation zone }\end{array}$ \\
\hline CT pulse & $\begin{array}{c}\text { Comp. zone precedes } \\
\text { Tens. zone }\end{array}$ & & & TC pulse \\
\hline
\end{tabular}

TABLE III: Explanation of nonreciprocal wave propagation in an asymmetric bilinear chain.

For current 2D monatomic lattice, an incident wave is generated by a continuous excitation in the linear section. When the incidence enters the nonlinear section consisting of multiple asymmetric bilinear chains connected together, it becomes a form with multiple cycles of two types of pulses, TC and CT one. After going through the similar transmissions shown in Fig. 7, e.g., (a) for incidence from position A and (b) for that from B, the transmitted waves with displacements in the opposite directions then scatter into the linear section again and detected by the receiver.

\section{Amplitude independence}

The nonreciprocal phenomenon of the opposite-sign dynamic profiles is presumably independent of input excitation amplitude given that the bilinear springs in the 2D lattice 
are only sensitive to the sign of the relative wave motion between unit cells connected to them. Here we apply the continuous excitation with several different amplitudes to show the amplitude-independent property.

We first set excitation amplitude positive, $U>0$, as in previous demonstrations. Figure 8(a) shows that the resultant dynamic profile scales linearly with the input amplitude, so that the normalized results overlap and therefore amplitude independence holds. Setting the excitation amplitude negative, $U<0$, shows the same scaling and amplitude independence, shown in Fig. 8(b); however the dynamic profiles are different from those of positive $U$. The reason is that the opposite signs of excitation amplitudes lead to different pulse orders within each cycle of the incident wave: a CT pulse precedes a TC one for the negative-value case; the reverse condition, TC before CT, is the case of positive excitation amplitude. Since they are fundamentally different inputs, this phenomenon does not violate the amplitudeindependent property.
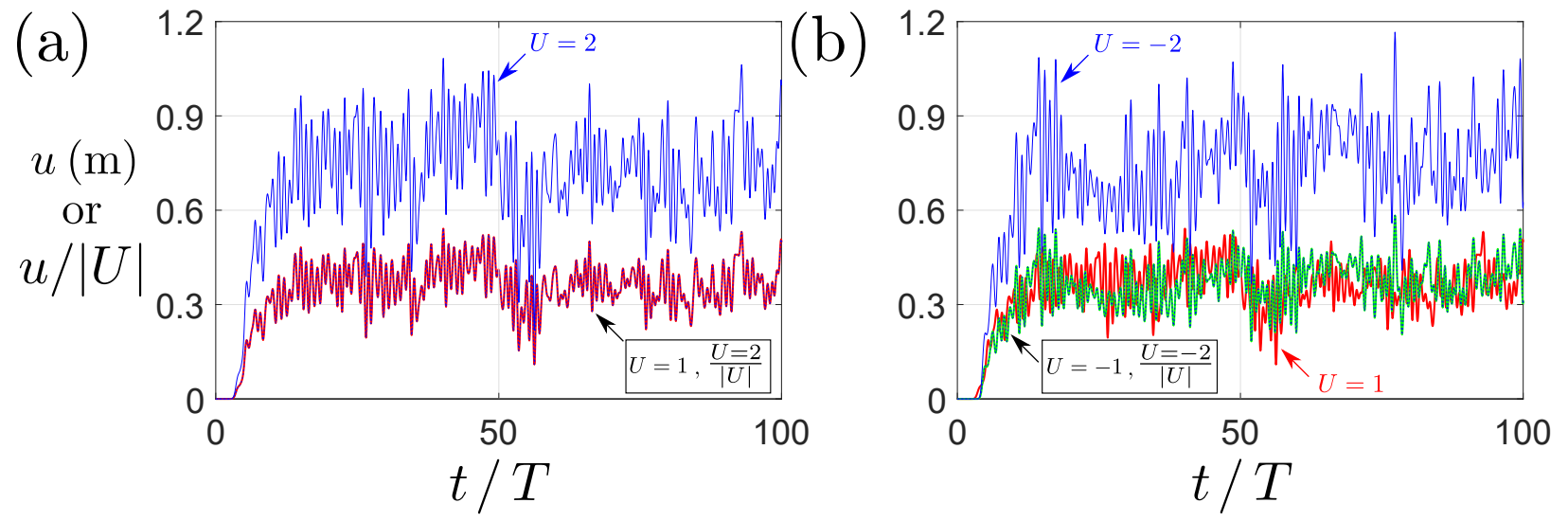

FIG. 8: Amplitude-independence. Simulations are performed in the setup with asymmetric bilinear arrangement along horizontal direction only in the nonlinear section, refer to Fig. 6(e), and the dynamic profiles of receiver at position B are recorded. (a) shows the results when we set positive excitation amplitudes $U=1$ and $2 \mathrm{~m}$, see Eq. (13); the overlapping normalized results demonstrate the amplitude-independent property. (b) shows the results with amplitudes $U= \pm 1$ and $-2 \mathrm{~m}$; the normalization of all negative-amplitude profiles are overlapped; however, because changing the sign of amplitude leads to a different incident wave, the opposite signs of excitation amplitudes result in different normalized dynamic behaviors. 


\section{Alternative modulation arrangements}

We display two more nonreciprocal demonstrations applying different linear and nonlinear section setups to show the micro-modulation design's capability of breaking reciprocity.

\section{Bilinearity + spatial asymmetry in both horizontal and vertical directions.}

Here we arrange the combination of bilinearity and spatial asymmetry in both horizontal and vertical directions, and set the positions for locating source and receiver are A, B and $\mathrm{C}$ as Fig. 9(a) shows. Dynamic profiles with the opposite amplitudes can be observed when considering the positions $\mathrm{A}$ and $\mathrm{B}$, but the amplitude of the negative profile decreases approaching to zero, see Fig. 9(b). For case of considering positions B and C where the receiver can presumably detect the signal coming from the source in linear condition, we can also obtain the dynamic profiles with opposite shifts as Fig. 9. (c) shows.
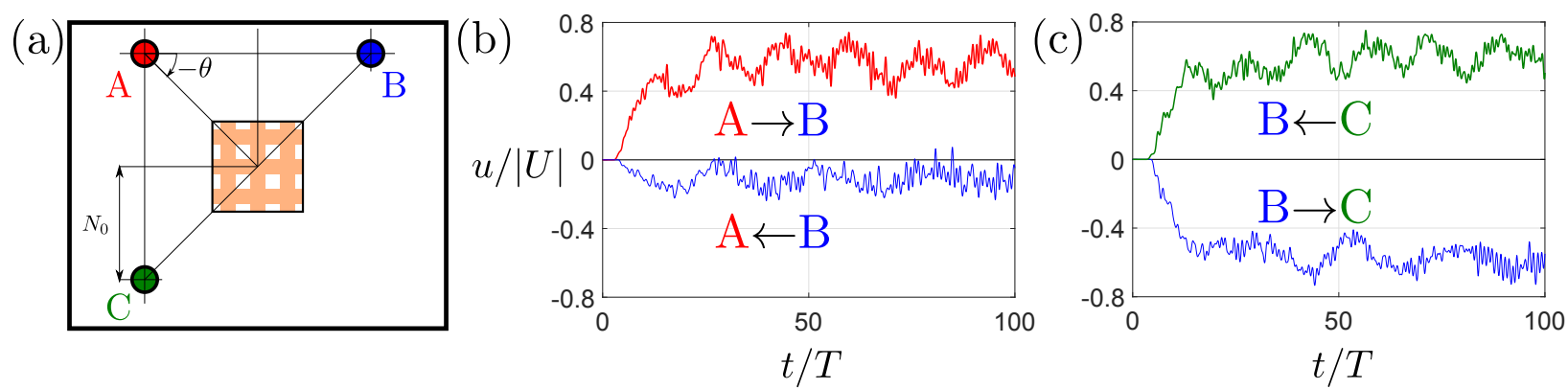

FIG. 9: Bilinearity + spatial asymmetry in both horizontal and vertical directions. (a) shows the simulation setup. The combination of bilinearity and spatial asymmetry is arranged in both horizontal and vertical directions. Three positions A, B and C are used to locate source and receiver; $N_{0}=10$ for position C. (b) depicts the different dynamic behaviors with decreasing negative shift for incidence from position B compared with Fig. 6(f). (c) shows the dynamic profiles with the opposite signs when positions B and C are considered.

Broadband effect. Our nonreciprocal design works for any case of the receiver being located in the propagation-free region relative to the source. Here we set $\kappa_{1}=1 \mathrm{~N} / \mathrm{m}$, $\kappa_{2}=1.5 \mathrm{~N} / \mathrm{m}$ for linear springs and keep the nonlinear section and excitation unchanged, which results in a directional wave propagation with ranges $\theta_{\text {range }} \approx 102^{\circ}$ instead of several discrete directions in previous demonstrations, see Fig. 10(a) and (b). As Fig. 10(c) shows, we can still get the nonreciprocal dynamic profiles with the opposite signs before and after interchanging the positions of source and receiver. 

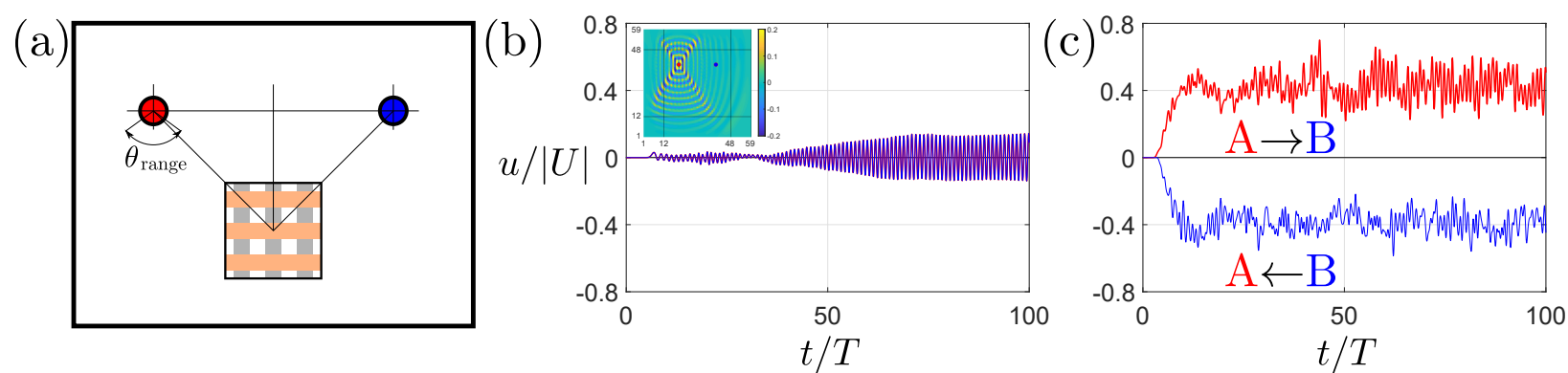

FIG. 10: Broadband effect. (a) shows the directional wave propagation covering a range with $\theta_{\text {range }} \approx 102^{\circ}$. (b) shows the dynamic profiles in the reciprocal linear case; the insert plot intuitively depicts the propagating ranges. (c) shows the nonreciprocal dynamic behaviors with the opposite signs of displacements before and after interchanging the positions of source and receiver.

\section{MACRO-MODULATION: SPATIAL ASYMMETRY OF BILINEAR SECTION}

Micro-modulation within the unit cell is a fundamental way of generating spatial asymmetry. With the source and receiver locations fixed, the whole bilinear section can be modulated in its entirety to further generate additional spatial asymmetry.

\section{Modulation beyond the unit cell}

In addition to generating the spatial asymmetry within the unit cell, we can extend the spatial modulation at a larger scale. When the positions of source and receiver are fixed, the modification of the whole bilinear section in its entirety can generate additional spatial asymmetry in a macro perspective. However, simply performing the macro-modulation for the whole bilinear section without the micro-modulation in its unit cells is proved to be an inefficient way of breaking reciprocity. Adding additional spatial asymmetry into the micro-modulated bilinear section is able to adjust the nonreciprocal results.

Here, we use the demonstrated nonreciprocal case of bilinearity and spatial asymmetry existing in both horizontal and vertical directions, see Fig. 9, in Sect. IV, and introduce additional macro-modulations of spatial asymmetry in the whole bilinear section to check their influences on nonreciprocity. We only take the positions A and B for tests, and the same excitation is applied, see Eq. (13). 


\section{Horizontal translation}

We fix the positions of source and receiver, but displace the whole bilinear section laterally by $\Delta N$ number of springs from its original position, as Fig. 11(a) shows. Simulation results are presented in Fig. 11(b); in stead of the whole dynamic profiles, only the maximum amplitudes are recorded; the ratios of two maximum amplitudes before and after interchanging the positions of source and receiver for each value of $\Delta N$ is also calculated, cases closer to -1 being treated as the more significantly nonreciprocal results. The lateral translation to the left, $\Delta N<0$, results in two dynamic profiles with the same positive sign and the amplitude ratio approaching to +1 , weakening the nonreciprocal behavior.
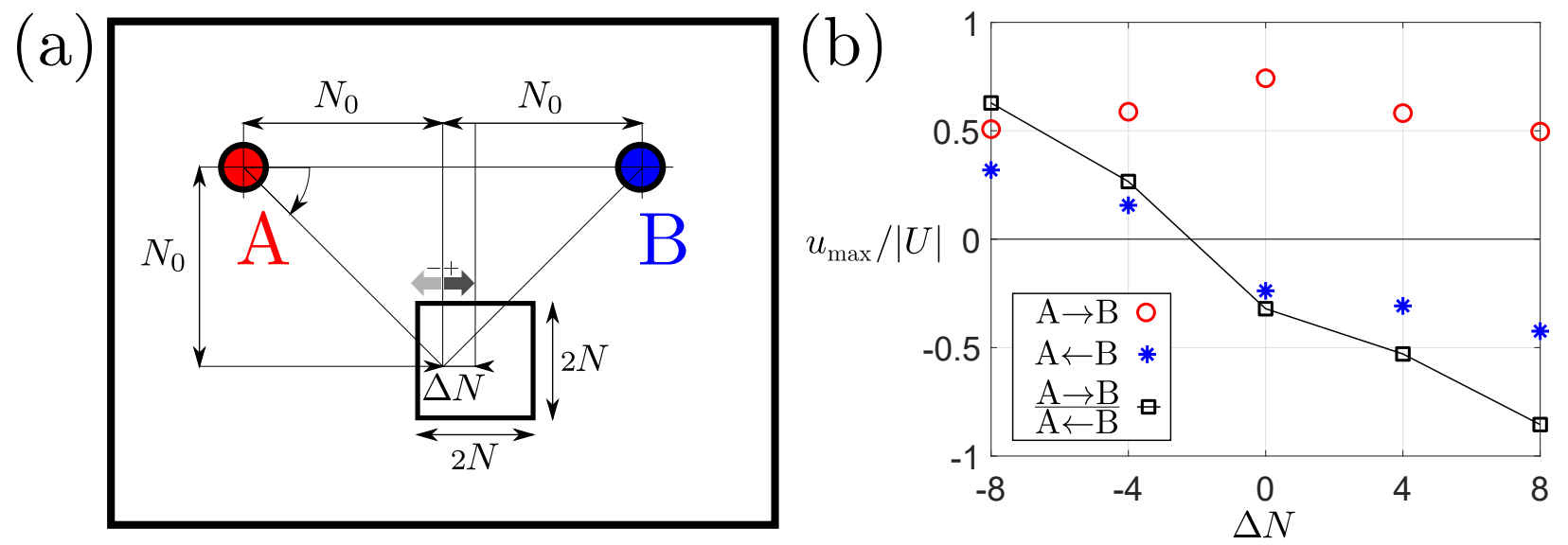

FIG. 11: Horizontal translation. (a) shows the horizontal translation of the bilinear section from its original position with positive $\Delta N$ the distance (number of springs) moved to the right; $N_{0}=$ $10, N=8$. (b) denotes the effect that value $\Delta N$ has on nonreciprocal behavior, which is indicated by the maximum amplitudes of dynamic profiles at two receivers and the ratio between them; the right translation results in the ratio approaching to -1, meaning the exaggeration of nonreciprocity; the left one leads to the same amplitude sign, weakening the nonreciprocity.

\section{Shape deformation}

Another macro-modulation fixes the center of the bilinear section at its original position and deforms its previous square shape into a parallelogram. In Fig. 12(a), $\gamma$ describes the angle by which the vertical boundaries of the bilinear section tilt. Namely, we displace the bilinear arrangements laterally by $n \tan \gamma, n=1,2, \ldots, N$ from the center of bilinear section 

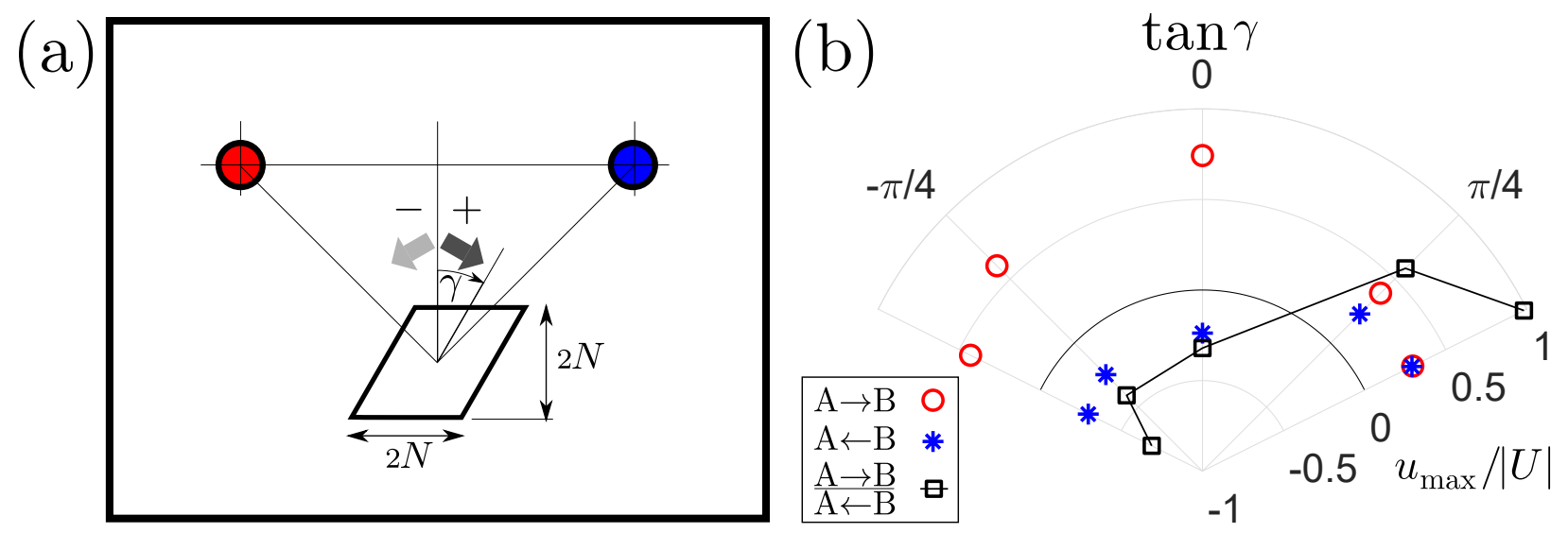

FIG. 12: Shape deformation. (a) depicts the shape deformation of the bilinear section into a parallelogram with vertical boundaries tilted clockwise by positive angle $\gamma$; this demonstration uses the same parameters as that in Fig. 11(a). (b) denotes the influence of tilting angle $\gamma$, labeled by $\tan \gamma$ in the polar plot; the maximum amplitudes of dynamic profiles at two receivers and their ratio for each value of $\gamma$ are used for checking; the modulations of the bilinear section can either weaken (clockwise tilting) or exaggerate (counterclockwise tilting) the nonreciprocity.

to the top for the upper half, and $-n \tan \gamma$ from the center to the bottom for the lower half. Figure 12(b) depicts the effect of the shape modulation, showing that clockwise tilt, $\gamma$ or $\tan \gamma>0$, weakens the nonreciprocal behaviors, but counterclockwise tilting, $\gamma$ or $\tan \gamma<0$, exaggerates the nonreciprocity.

\section{CONCLUSION}

Taking advantage of the directional nature of wave propagation in the 2D monatomic lattice, we have demonstrated a passive approach to breaking reciprocity via the introduction of a bilinear section with spatially asymmetric properties, which displays amplitudeindependent nonreciprocal scattering. Utilizing the 2D linear anisotropic wave properties of the lattice, the source and receiver are chosen to be positioned so that the direct linear signal approximates zero and therefore the asymmetric nonlinearity-induced scattered signals with opposite signs dominate. The 2D lattice configuration opens up the possibility of spatially asymmetric configurations at different scales. The micro-modulations, either by bilinear stiffness alteration or by physical structure modification, within each unit cell of the nonlinear section fundamentally generate the spatial asymmetry. Additional macro-modulations 
aiming at the whole bilinear section in its entirety, for example by lateral displacement or by deformation of section's shape, can further weaken or exaggerate the existing nonreciprocal behavior. Moreover, different simulation setups resulting in directional propagation and nonreciprocity prove that our design has the capability of breaking reciprocity as long as a propagation-free zone exists.

\section{Acknowledgment}

This work is supported by the NSF EFRI Program under Award No. 1641078.

\section{Appendix A: Perfectly matched layers}

To simulate a nonreflecting infinite transverse wave, perfectly matched layers (PMLs) are attached to the sides and corners of the 2D monatomic lattice, as Fig. 13 shows. The PML is a damped monatomic lattice with ramped-up damping coefficients to avoid reflections caused at the interfere of damped and non-damped sections. The varying damping coefficients can be expressed as

$$
C_{j}=C_{\max }\left(\frac{j}{N_{P M L}}\right)^{3},
$$

where $C_{j}$ represents the damping coefficient at index $j$ that starts from 1 at the interfere of PML and non-damped lattice, $C_{\max }$ is the maximum damping coefficient located at the end of the PML, and $N_{P M L}$ denotes the the number of dampers per column for the upper and lower PMLs or per row for the left and right PMLs in Fig. 13. The damping coefficients in PMLs at four corners are more complicated to be expressed but also obey the rule of values increasing from interfere to boundaries. Here we take $N_{P M L}=10$ and $C_{\max }=10 \mathrm{~kg} \cdot \mathrm{s}^{-1}$ for all numerical simulations in this paper. 


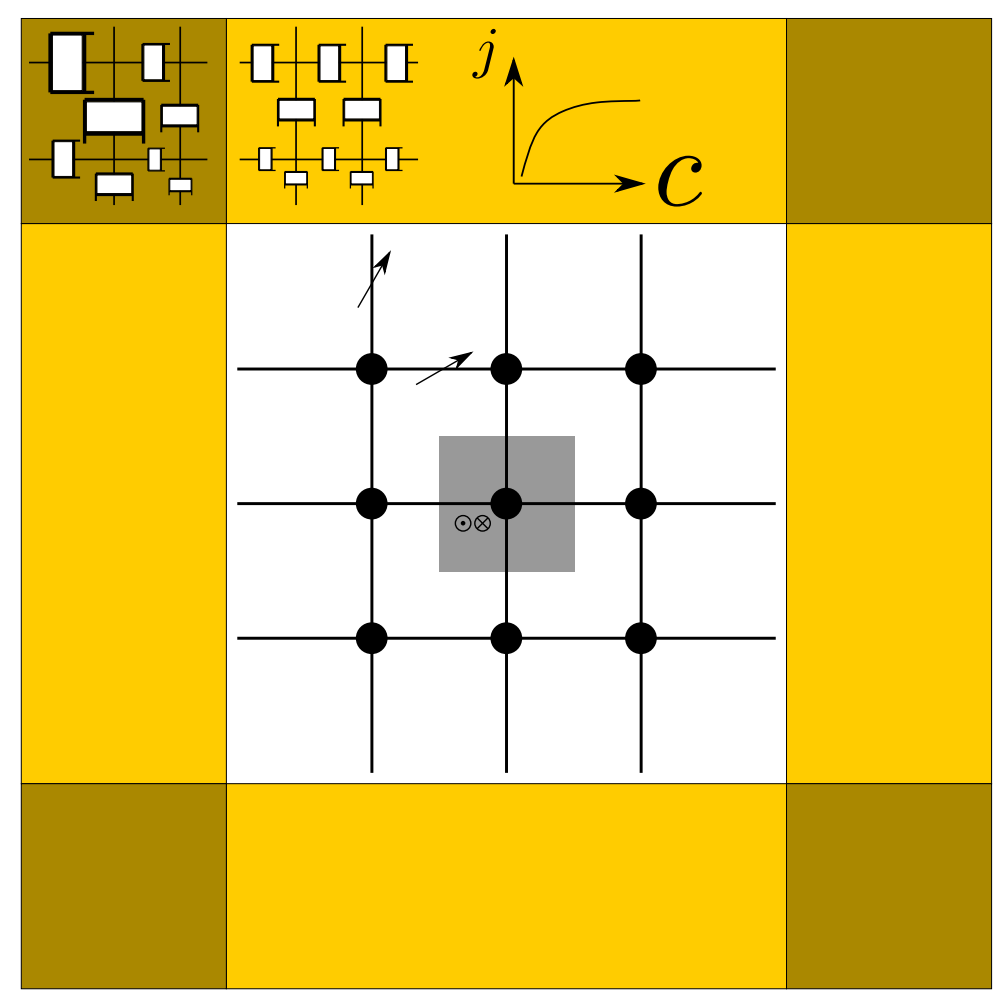

FIG. 13: Perfectly matched layers (PMLs) surround the 2D monatomic lattice. PML is a damped monatomic lattice with damping coefficients ramping up from the interfere of damped and nondamped lattice to the boundary. The size of damping cartoon in PMLs denotes the value of damping coefficient, the larger the size the greater the value.

\section{Data availability}

Simulation data were generated by ODE solver in MATLAB. The datasets generated during and/or analysed during the current study are available from the corresponding author on reasonable request.

\section{Conflict of Interest}

The authors declare that they have no conflict of interest. 
[1] Hussein Nassar, Behrooz Yousefzadeh, Romain Fleury, Massimo Ruzzene, Andrea Alù, Chiara Daraio, Andrew N. Norris, Guoliang Huang, and Michael R. Haberman. Nonreciprocity in acoustic and elastic materials. Nature Reviews Materials, 5(9):667-685, jul 2020.

[2] Romain Fleury, Dimitrios L Sounas, Caleb F Sieck, Michael R Haberman, and Andrea Alù. Sound isolation and giant linear nonreciprocity in a compact acoustic circulator. Science, 343(6170):516-519, 2014.

[3] Farzad Zangeneh-Nejad and Romain Fleury. Doppler-based acoustic gyrator. Applied Sciences, 8(7):1083, jul 2018.

[4] G. Trainiti and M. Ruzzene. Non-reciprocal elastic wave propagation in spatiotemporal periodic structures. New J. Phys., 18(8):083047, 2016.

[5] H. Nassar, H. Chen, A. N. Norris, M. R. Haberman, and G. L. Huang. Non-reciprocal wave propagation in modulated elastic metamaterials. Proceedings of the Royal Society A: Mathematical, Physical and Engineering Sciences, 473(2202):20170188, jun 2017.

[6] H. Nassar, H. Chen, A.N. Norris, and G.L. Huang. Non-reciprocal flexural wave propagation in a modulated metabeam. Extreme Mechanics Letters, 15:97-102, sep 2017.

[7] H. Nassar, X.C. Xu, A.N. Norris, and G.L. Huang. Modulated phononic crystals: Nonreciprocal wave propagation and Willis materials. Journal of the Mechanics and Physics of Solids, 101:10-29, 2017.

[8] Yifan Wang, Behrooz Yousefzadeh, Hui Chen, Hussein Nassar, Guoliang Huang, and Chiara Daraio. Observation of nonreciprocal wave propagation in a dynamic phononic lattice. Physical Review Letters, 121(19), nov 2018.

[9] Benjamin M. Goldsberry, Samuel P. Wallen, and Michael R. Haberman. Non-reciprocal wave propagation in mechanically-modulated continuous elastic metamaterials. The Journal of the Acoustical Society of America, 146(1):782-788, jul 2019.

[10] Benjamin M. Goldsberry, Samuel P. Wallen, and Michael R. Haberman. Nonreciprocal vibrations of finite elastic structures with spatiotemporally modulated material properties. Physical Review B, 102(1), jul 2020.

[11] Samuel P. Wallen, Michael R. Haberman, Zhaocheng Lu, Andrew Norris, Tyler Wiest, and Carolyn C. Seepersad. Static and dynamic non-reciprocity in bi-linear structures. In Proceed- 
ings of Meetings on Acoustics 21ISNA. Acoustical Society of America, 2018.

[12] Itay Grinberg, Alexander F. Vakakis, and Oleg V. Gendelman. Acoustic diode: Wave nonreciprocity in nonlinearly coupled waveguides. Wave Motion, 83:49-66, dec 2018.

[13] Keegan J. Moore, Jonathan Bunyan, Sameh Tawfick, Oleg V. Gendelman, Shuangbao Li, Michael Leamy, and Alexander F. Vakakis. Nonreciprocity in the dynamics of coupled oscillators with nonlinearity, asymmetry, and scale hierarchy. Physical Review E, 97(1), jan 2018.

[14] Qifan Zhang, Wei Li, John Lambros, Lawrence A. Bergman, and Alexander F. Vakakis. Pulse transmission and acoustic non-reciprocity in a granular channel with symmetry-breaking clearances. Granular Matter, 22(1), dec 2019.

[15] Zhaocheng Lu and Andrew N. Norris. Non-reciprocal wave transmission in a bilinear springmass system. Journal of Vibration and Acoustics, 142(2), dec 2019.

[16] Zhaocheng Lu and Andrew N. Norris. Unilateral and nonreciprocal transmission through bilinear spring systems. Extreme Mechanics Letters, 42:101087, jan 2021.

[17] Lezheng Fang, Alireza Mojahed, Amir Darabi, Alexander F. Vakakis, and Michael J. Leamy. Passive nonreciprocity in a system of asymmetrical rotational oscillators. Physical Review Applied, 15(3), mar 2021.

[18] Amir Darabi, Lezheng Fang, Alireza Mojahed, Matthew D. Fronk, Alexander F. Vakakis, and Michael J. Leamy. Broadband passive nonlinear acoustic diode. Physical Review B, 99(21), jun 2019.

[19] Lisa M. Nash, Dustin Kleckner, Alismari Read, Vincenzo Vitelli, Ari M. Turner, and William T. M. Irvine. Topological mechanics of gyroscopic metamaterials. Proceedings of the National Academy of Sciences, 112(47):14495-14500, nov 2015.

[20] Alexander B. Khanikaev, Romain Fleury, S. Hossein Mousavi, and Andrea Alù. Topologically robust sound propagation in an angular-momentum-biased graphene-like resonator lattice. Nature Communications, 6(1), oct 2015.

[21] Pai Wang, Ling Lu, and Katia Bertoldi. Topological phononic crystals with one-way elastic edge waves. Physical Review Letters, 115(10), sep 2015.

[22] Qian Wu, Hui Chen, Hussein Nassar, and Guoliang Huang. Non-reciprocal rayleigh wave propagation in space-time modulated surface. Journal of the Mechanics and Physics of Solids, 146:104196, jan 2021. 
[23] Massimo Ruzzene, Fabrizio Scarpa, and Francesco Soranna. Wave beaming effects in twodimensional cellular structures. Smart Materials and Structures, 12(3):363-372, apr 2003.

[24] A. Spadoni, M. Ruzzene, S. Gonella, and F. Scarpa. Phononic properties of hexagonal chiral lattices. Wave Motion, 46(7):435-450, 2009.

[25] R. K. Narisetti, M. Ruzzene, and M. J. Leamy. A perturbation approach for analyzing dispersion and group velocities in two-dimensional nonlinear periodic lattices. Journal of Vibration and Acoustics, 133(6), dec 2011. 